\title{
Surgery Insight: the role of surgery in the management of low-grade gliomas
}

\author{
Nader Pouratian, Ashok Asthagiri, Jay Jagannathan, Mark E Shaffrey and David Schiff*
}

\section{SUMMARY}

The benefits of surgery for the management of low-grade gliomas have been difficult to determine from the literature. This difficulty might be explained by the inconsistency of the published data, and also by advances in both neuroimaging and neurosurgical techniques, which have made surgical intervention a safer and more viable option than it has been in the past, making the earlier studies less applicable to modern care. In this article, we critically analyze the utility of surgery in the management of low-grade gliomas, including the value of observation without surgical intervention, the relative risks and benefits of biopsy versus craniotomy and resection, and recent advances that have made surgery safer and gross total resection a more realistic proposition. As we will discuss, the literature provides modest evidence that surgery leads to improved outcomes through a reduction in tumor burden. As a result of advances in surgical techniques, the time might now be right to effectively and accurately assess the influence of aggressive surgical resection on the prognosis of low-grade gliomas.

KEYWORDS astrocytoma, low-grade glioma, oligoastrocytoma, oligodendroglioma, surgical resection

\section{REVIEW CRITERIA}

For this review, we searched PubMed by using Entrez without date restrictions and including early release publications. Search terms included "low-grade glioma" in combination with each of the following terms: "observation", "biopsy" and "surgery". The abstracts of retrieved citations were reviewed for relevant content. Full articles were obtained and references were checked for additional material when appropriate.

\section{CME}

N Pouratian is a Neurosurgical Resident, J Jagannathan is a Resident in Neurosurgery, ME Shaffrey is Professor and Chairman of Neurosurgery, and $D$ Schiff is a Medical Neuro-Oncologist and Professor, all at the University of Virginia, Charlottesville, VA, USA. A Asthagiri is a Staff Clinician at the NIH, Bethesda, MD, USA.

\section{Correspondence}

*Neuro-Oncology Center, University of Virginia Health System, PO Box 800432, Charlottesville, VA 22908-0432, USA

davidschiff@virginia.edu

Received 14 June 2007 Accepted 9 August 2007

www.nature.com/clinicalpractice

doi:10.1038/ncpneuro0634
Medscape Continuing Medical Education online Medscape, LLC is pleased to provide online continuing medical education (CME) for this journal article, allowing clinicians the opportunity to earn CME credit. Medscape, LLC is accredited by the Accreditation Council for Continuing Medical Education (ACCME) to provide CME for physicians. Medscape, LLC designates this educational activity for a maximum of 1.0 AMA PRA Category 1 Credits $^{T M}$. Physicians should only claim credit commensurate with the extent of their participation in the activity. All other clinicians completing this activity will be issued a certificate of participation. To receive credit, please go to http://www.medscape.com/cme/ncp and complete the post-test.

\section{Learning objectives}

Upon completion of this activity, participants should be able to:

1 Distinguish types of low-grade gliomas.

2 Describe the prevalence of low-grade gliomas.

3 Identify the most common presentations of lowgrade gliomas.

4 Describe characteristics of low-grade gliomas amenable to surgical resection.

5 List potential benefits of gross total resection for patients with low-grade gliomas.

\section{INTRODUCTION}

Although their name might imply otherwise, low-grade gliomas result in considerable morbidity and inevitable death. The benefits of various interventions-including fractionated radiotherapy, chemotherapy and surgical resection-have all been disputed, but the role of surgical intervention is particularly controversial. This debate stems in part from the inconsistency of results from published reports regarding the benefits of surgical intervention, and is complicated further by advances in neuroimaging and neurosurgical techniques that have made surgical resection a safe and viable option for an increasing number of patients.

In this Review, we will critically analyze the utility of surgery in the management of low-grade gliomas, including the role of observation without surgical intervention, the debate regarding biopsy versus resection, and the recent surgical advances that have made surgery for low-grade gliomas increasingly safe and widely available. 


\section{EPIDEMIOLOGY}

The phrase 'low-grade gliomas' encompasses the entire spectrum of WHO grade I and II gliomas, which includes ependymomas, pilocytic astrocytomas, pleomorphic xanthoastrocytomas, diffuse astrocytomas, oligodendrogliomas, and mixed gliomas. Although arbitrarily clustered under the umbrella low-grade glioma nomenclature, this heterogeneous group of tumors is clinically, histologically and molecularly diverse, and is, therefore, not generally studied or discussed as a single entity. For example, WHO grade I lesions, which include pilocytic astrocytomas and gangliogliomas, are, unlike WHO grade II lesions, well circumscribed and noninfiltrative, and complete surgical resection is universally considered curative. ${ }^{1}$ In this Review, we will limit our discussion to WHO grade II diffuse astrocytomas, oligodendrogliomas and oligoastrocytomas, all of which have similar invasive and malignant potential.

Although low-grade gliomas are less common than malignant gliomas (of which there are $\sim 8,000-10,000$ cases per year) and far less common than brain metastases $(\sim 75,000$ 150,000 cases per year), they are far from rare. Approximately 2,000-3,000 low-grade gliomas are diagnosed in the US every year, accounting for nearly $15 \%$ of all primary brain tumors. ${ }^{2}$ According to the Central Brain Tumor Registry of the United States (CBTRUS), the various histological classes of low-grade glioma have incidences varying between 0.10 and 0.46 per 100,000 people, and a cumulative incidence of approximately 0.9 per 100,000 people. $^{2}$

Despite the preponderance of astrocytomas, there has been an apparent increase in recent years in the incidence of pure oligodendrogliomas and of mixed oligoastrocytomas. This increase might be partly attributable to an increased tendency for neuropathologists to seek out such diagnoses, in view of the relatively favorable prognosis conveyed by an oligodendroglial component. ${ }^{3}$ Low-grade gliomas are particularly prevalent among white people and among men, and the highest incidence is in people between 35 and 44 years of age. Low-grade astrocytomas in adults are most commonly located in the cerebral hemispheres, with a predilection for 'secondary' functional areas such as the supplementary motor area and the insular lobe. ${ }^{4}$ Oligodendrogliomas are most commonly seen along the cerebral convexity in subcortical areas, particularly in the frontal lobe, but sporadic reports of posterior fossa oligodendrogliomas exist.

\section{SIGNS, SYMPTOMS AND PROGNOSIS}

Low-grade gliomas produce signs and symptoms of disease through three main mechanisms: direct infiltration and destruction of neurons; local pressure from edema, hemorrhage and tumor mass; and intracranial hypertension owing to mass effect or ventricular obstruction. In many cases, the insidious onset of symptoms related to low-grade gliomas might delay the correct diagnosis for many years. When patients become symptomatic, seizure is the most common presenting sign, occurring in up to $80 \%$ of cases. ${ }^{5,6}$ Other less common modes of presentation include headache, lethargy and personality changes. The patient's presenting symptoms and imaging characteristics can critically affect management; both neurosurgeons and neuro-oncologists are likely to recommend surgical intervention for patients presenting with signs and symptoms attributable to mass effect or intracranial hypertension.

Although the effect of surgery on low-grade glioma prognosis is a topic for debate, several factors are recognized to be associated with poor prognosis, including, but not limited to, advanced age at time of diagnosis, ${ }^{3,5-7}$ larger tumor size on presentation (before surgical intervention), ${ }^{6,8-10}$ and rapid tumor growth rate. ${ }^{11}$ Favorable prognostic factors include presentation with seizure (as opposed to with an abnormal neurological examination), and having an oligodendroglial component, a $1 \mathrm{p}$ and $19 \mathrm{q}$ chromosomal deletion, or a tumor that does not cross the midline.

\section{IS THERE A ROLE FOR OBSERVATION WITHOUT SURGERY?}

Historically, expectant management has been considered to be a plausible management paradigm for patients who have lesions with typical features of low-grade glioma, especially those with minimal symptoms or seizures that are well controlled with antiepileptic drugs. ${ }^{12}$ Expectant management can potentially provide patients with an overall improved quality of life (QOL) for the duration of disease by not exposing them at the time of diagnosis to the morbidity and mortality risks associated with biopsy or with craniotomy and resection. Imaging characteristics considered to be typical of low-grade glioma include low attenuation on 


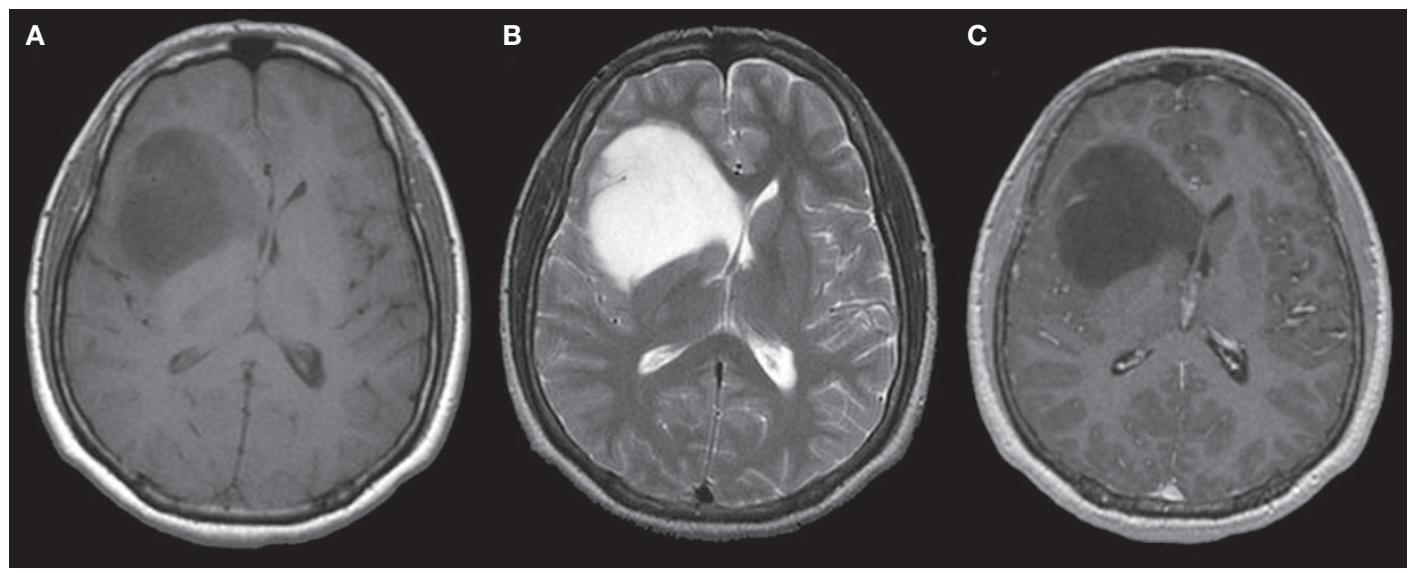

Figure 1 Typical MRI scan of a low-grade glioma, histopathologically defined as a WHO grade II oligodendroglioma. (A) T1 sequence demonstrating T1 shortening in the right frontal lobe. (B) T2 sequence demonstrating T2 prolongation (hyperintensity) at the site of the glioma. (C) Contrast-enhanced imaging of the glioma showing no marked contrast enhancement. Although these images are considered 'typical', numerous studies have questioned the reliability and accuracy of these imaging characteristics for the diagnosis of low-grade glioma.

CT scanning, and T1 shortening, T2 prolongation, and absence of contrast enhancement on MRI (Figure 1). ${ }^{13}$ The diagnostic value of these 'typical' imaging characteristics has, however, come under scrutiny. Kondziolka and colleagues found that imaging-based diagnosis of low-grade glioma was accurate in only $50 \%$ of patients (on the basis of comparison with stereotactic biopsy results). ${ }^{13}$ Likewise, Scott and colleagues found that, in a series of 314 patients, grading gliomas on the basis of imaging characteristics alone underestimated the degree of malignancy in one-third of cases. ${ }^{14}$ Furthermore, although contrast enhancement on MRI has long been considered a sign of malignancy, recent studies of chemotherapeutics for low-grade glioma have reported contrast enhancement in up to $60 \%$ of low-grade gliomas. ${ }^{15}$ The use of magnetic resonance spectroscopy and perfusion-weighted imaging has markedly increased the accuracy and sensitivity of imaging-based diagnosis and grading of gliomas, but these techniques are still associated with significant error rates and are not used in routine clinical neuroimaging. ${ }^{16-18}$

The preponderance of studies that contradict the notion of a pathognomonic imaging profile for low-grade glioma that could be elucidated using standard imaging sequences suggests that tissue acquisition remains mandatory for the accurate diagnosis, prognostication and management of these tumors. In an era when management regimens are predicated on the basis of tumor cytogenetics, tissuebased diagnosis carries increasing prognostic and therapeutic implications and the absence of such evaluation inevitably limits the scope of therapeutic interventions.

In addition to misdiagnosis, expectant management carries other risks, including malignant degeneration, interval tumor growth that makes subsequent resection more difficult or impossible, and development of an irreversible neurological deficit. ${ }^{19}$ Despite these theoretical risks, a comparison between initial conservative management and immediate surgical resection by Recht and colleagues reported that the timing of intervention did not affect rates of malignant transformation, overall survival (OS), or QOL. ${ }^{20}$ Similarly, Reijneveld and co-workers found that, although patients with low-grade gliomas experienced significant cognitive disturbances and impairments in QOL, a 'wait-and-see' policy did not worsen these afflictions. ${ }^{21}$ Furthermore, van Veelen et al. reported that the 5-year survival was identical $(63 \%)$ in patients who underwent surgery immediately on diagnosis and those who underwent surgery at the time of clinical or radiographic progression. ${ }^{22}$ Despite the consistent finding that the timing of tissue diagnosis is not critical, it is important to stress that the studies performed on this topic to date have all been retrospective, which inevitably introduces considerable selection bias and limits interpretation and applicability. 


\section{BIOPSY OR RESECTION?}

Both stereotactic biopsy and open biopsy provide an opportunity to characterize the histology and cytogenetics of a suspected low-grade glioma without the morbidity and mortality risks associated with a large craniotomy. In general, biopsy has been advocated in the setting of tumors located adjacent or deep to eloquent cortices, which would not be amenable to gross total resection (GTR). ${ }^{23}$ Whereas stereotactic biopsies are preferred for patients with deeper lesions, open biopsies are reserved for those with superficial lesions that are easily accessible via a small craniotomy and for those with lesions immediately adjacent to eloquent cortices, which should be mapped before biopsy. Craniotomy and resection, on the other hand, have traditionally been reserved for patients with appreciable mass effect from low-grade glioma (which results in increased intracranial pressure or neurological deficit) and for those with symptomatic epilepsy that is refractory to antiepileptic therapy. ${ }^{22,24}$

In general, the diagnostic yield of stereotactic biopsy is high (90-95\%). ${ }^{25-27}$ Several studies have, however, raised concerns regarding the reliability and accuracy of biopsy-based diagnoses. ${ }^{23,26,28,29}$ Aker and colleagues scrutinized results from 23 patients who initially underwent stereotactic biopsy and subsequently required craniotomy, and reported that the accuracy of histological diagnosis made on the basis of a stereotactic biopsy sample was only $83 \% .{ }^{26}$ In three of the four misdiagnosed cases, a biopsy-based diagnosis of low-grade glioma was changed to anaplastic astrocytoma, granulomatous cerebritis, or dysembryoblastic neuroepithelial tumor. Similarly, McGirt and co-workers reported $79 \%$ accuracy of diagnosis in 23 patients who underwent biopsy followed within 60 days by resection. ${ }^{28}$ Even more concerning, Jackson and colleagues at the MD Anderson Cancer Center, Houston, TX, reported accuracy rates inferior to those reported by McGirt et al., ranging between 51\% and $62 \% .{ }^{23}$ Accuracy rates depended on the expertise of the interpreting neuropathologists, highlighting the need for expert neuropathology consultation when using stereotactic biopsy in order to obtain the best possible accuracy. ${ }^{23}$ Despite discrepancies between histopathology results from stereotactic biopsy and craniotomy, some groups have argued that appropriate treatments are still administered in $93-96 \%$ of cases, owing to similarities in treatment paradigms for gliomas of different grades..$^{28,30}$ Such viewpoints are, however, becoming less valid as clinical studies further stratify treatment paradigms according to tumor grade and cytogenetics. Moreover, prognostication and patient counseling is inevitably adversely affected by misdiagnosis.

When assessing the value and role of biopsy in the management of low-grade gliomas, it is important to consider not only the yield and accuracy of biopsy, but also the risks associated with the procedure. Despite its reputation for safety, stereotactic biopsy is not without risk. To quantify the morbidity and mortality of the procedure, Hall reviewed 17 series comprising a total of 7,471 stereotactic brain biopsies. ${ }^{27}$ He reported an overall morbidity rate of 3.5\% and a mortality rate of $0.7 \%$, which were largely related to procedure-induced hemorrhage. Risk factors for biopsy-associated morbidity include basal ganglia lesions, thalamic lesions, diabetes, and hyperglycemia on the day of surgery. ${ }^{31}$

In contrast to biopsy, surgical resection minimizes the likelihood of sampling error by providing considerably more tissue for histopathological analysis, but it inherently exposes the patient to greater risk. Enthusiasm for surgical resection has grown in light of advances in neuroimaging and neurosurgical techniques that have made surgical resection safer and more accessible, and on the basis of numerous reports on the benefits of GTR with respect to both symptom control and prolongation of survival and time to progression. Although not curative, GTR theoretically provides multiple benefits, including reduction in the number of cells at risk of undergoing genetic events that result in malignant transformation, increased efficacy of adjuvant therapy, and symptomatic relief owing to reduced mass effect. Surgical resection has been particularly effective at controlling medically refractory seizures, resulting in near-seizure-free status, or a substantial reduction in seizure frequency and intensity, in nearly all patients. ${ }^{32,33}$

Although large preoperative tumor size (diameter $>5-6 \mathrm{~cm}$ ) is consistently identified as a poor prognostic factor for survival, ${ }^{3,6}$ the effect on clinical outcome of reduced tumor size remains controversial, and several studies have produced inconsistent results (Table 1). Keles and colleagues critically reviewed the literature with respect to the effect of extent of 
Table 1 Large studies investigating the effect of surgical resection on outcomes of low-grade glioma.

\begin{tabular}{|c|c|c|c|c|c|c|c|c|c|}
\hline \multirow[t]{2}{*}{ Reference } & \multirow[t]{2}{*}{$n$} & \multirow{2}{*}{$\begin{array}{l}\text { Types of } \\
\text { low-grade } \\
\text { glioma }\end{array}$} & \multirow[t]{2}{*}{ Study type } & \multirow{2}{*}{$\begin{array}{l}\text { Assessment } \\
\text { of extent } \\
\text { of tumor } \\
\text { resection }\end{array}$} & \multirow{2}{*}{$\begin{array}{l}\text { Surgical } \\
\text { selection } \\
\text { criteria } \\
\text { described }^{c}\end{array}$} & \multirow{2}{*}{$\begin{array}{l}\text { Use of } \\
\text { multivariate } \\
\text { analysis used }^{\text {d }}\end{array}$} & \multicolumn{3}{|c|}{$\begin{array}{l}\text { Extensive resection } \\
\text { statistically improved }\end{array}$} \\
\hline & & & & & & & os & PFS & QOL \\
\hline Shaw et al. $(1989)^{72}$ & 126 & $A, M$ & Retrospective & Surgeon & No & Yes & No & No & NS \\
\hline Philippon et al. $(1993)^{73}$ & 179 & $A$ & Retrospective & Surgeon & No & Yes & Yes & NS & NS \\
\hline Karim et al. $(1996)^{10}$ & 343 & $\mathrm{~A}, \mathrm{O}, \mathrm{M}$ & Retrospective & Surgeon & No & Yes & Yes & Yes & NS \\
\hline Scerrati et al. (1996) $)^{75}$ & 131 & $\mathrm{~A}, \mathrm{O}, \mathrm{M}$ & Retrospective & Surgeon & No & Yes & Yes & NS & NS \\
\hline Leighton et al. (1997) ${ }^{7}$ & 167 & $\mathrm{~A}, \mathrm{O}, \mathrm{M}$ & Retrospective & Surgeon & No & Yes & Yes & Yes & NS \\
\hline Lote et al. $(1997)^{37}$ & 379 & $\mathrm{~A}, \mathrm{O}, \mathrm{M}$ & Retrospective & Surgeon & No & No & No & No & NS \\
\hline
\end{tabular}

aOnly studies including at least 100 adult patients with supratentorial low-grade glioma (including astrocytomas, oligoastrocytomas and mixed gliomas) are featured in this table. ' Surgeon= primary surgeon's impression. 'Whether the report adequately described the indications for surgical resection and whether the series included consecutive patients ${ }^{d}$ Whether the report controlled for possible covariates by using multivariate analysis esignificant effect seen on univariate analysis, but not on multivariate analysis. Abbreviations: A, astrocytoma; M, mixed glioma; $n$, number of patients studied; ND, not described in the paper; NS, not studied; O, oligodendroglioma; OS, overall survival; PFS, progression-free survival; QOL, quality of life.

resection on outcomes in low-grade glioma and found that a preponderance of modern studies support extensive resection over biopsy alone. ${ }^{19}$ Leighton and co-workers, who retrospectively studied 167 consecutive patients treated at the London Regional Cancer Centre, ON, Canada, for low-grade glioma over a period of 16 years found, on both univariate and multivariate analysis, that minimal postoperative residual tumor was associated with significantly improved 5-year OS ( $82 \%$ vs $64 \% ; P=0.008$ on univariate analysis, $P=0.006$ on multivariate analysis). ${ }^{7}$ Other groups have likewise reported, in retrospective series, that aggressive surgical resection can prolong the time to tumor recurrence and reduce the malignant transformation rate. ${ }^{5,8,34}$ Lo and colleagues specifically queried whether patients who received postoperative radiation therapy had benefited from extensive resections. ${ }^{35}$ Of the 65 patients who received postoperative radiation therapy, the 12 patients who had GTR (as assessed by postoperative imaging) had significantly longer 10-year OS than those who had subtotal resection or biopsy only ( $90 \%$ vs $41.4 \% ; P=0.001)$.

The results from prospective trials on resection and postoperative radiation therapy, however, are not so compelling. For example, a prospective intergroup American trial, designed primarily to compare the effects of low-dose versus high-dose radiation therapy, reported, on univariate analysis, a significant 5-year OS advantage in patients who had GTR compared with those who had subtotal resection or biopsy alone $(88 \%$ vs $56 \%$ and $71 \%$, respectively; $P=0.0151){ }^{3}$ This trial also reported prolonged progression-free survival (PFS) in patients who had GTR compared with those who did not (hazard ratio $0.44, P=0.0138$ on univariate analysis). ${ }^{3}$ Pignatti and colleagues and Karim and co-workers both retrospectively studied prospectively collected data from European Organisation for Research and Treatment of Cancer trials (22844 and 22845, and 22844 only, respectively) and found, on univariate analysis, that extensive resection (i.e. $>90 \%$ ) was associated with longer OS. 6,10 Despite consistent findings on univariate analysis, on multivariate analysis the benefits of extensive resection were marginal at best in both studies. The failure of prospective trials to identify a benefit on multivariate analysis seriously challenges the role of extensive surgery in the management of low-grade gliomas. It is important to point out, however, that these studies were not primarily intended to assess the effect of extensive resection, and were, therefore, not 
designed or powered to reliably evaluate this factor. For example, in most studies, the extent of resection was gauged on the basis of the 'surgeon's impression', which is an unreliable assessment. Further complicating the interpretation of the literature are several other studies that contradict the finding that the difference in OS or PFS among patients relates to the extent of resection. ${ }^{19,36,37}$

The effect of surgical resection on QOL, as well as on survival, must be considered. Teixidor and colleagues recently studied the consequence of surgical resection on higher cognitive functions, specifically verbal working memory, as this function can dramatically affect a patient's QOL. ${ }^{38}$ As in previous studies, the authors found that most patients with low-grade glioma had preoperative cognitive deficits. ${ }^{21,38}$ Although surgery can induce further deficits, these exacerbations are transient in most patients. Patients generally recover preoperative cognitive function, or even improve upon it, within 3 months after surgery.

Despite the preponderance of modern studies favoring GTR, there is a paucity of class I evidence supporting or refuting radical tumor removal. Moreover, the available statistical evidence in both retrospective and prospective reports published to date is adulterated by numerous inherent limitations, including patient-selection bias, treatment-selection bias, and inconsistent and inaccurate means of assessing the extent of resection. The effect of selection bias in particular should not be underestimated. Unfortunately, surgical selection criteria are rarely, if ever, reported (Table 1). Patients with polar or lobar tumors with sharp borders are more likely to undergo and benefit from aggressive resection than those with less well defined lesions. By contrast, patients with probable low-grade glioma who are fully functional and never undergo treatment are not enrolled in surgical series. Tumor histopathology might also introduce selection bias; oligodendrogliomas - which often have sharp radiographic borders and are, therefore, more amenable to extensive resection than are fibrillary astrocytomas-have a better natural history than astrocytomas, and might thereby introduce a considerable potential selection bias into a surgical series. For each study, therefore, it is important to consider the patient and tumor characteristics of both patients who were included in the analysis and those who were excluded. Notwithstanding the effect of selection bias, the lack of a consistent means of measuring the extent of resection probably adversely affects the interpretation of the literature (Table 1). In some studies, the extent of resection has been assessed on the basis of the surgeon's impression alone, whereas in others it has been assessed by use of inferior imaging modalities such as CT or by objective evaluation of postoperative T2-weighted or fluidattenuated inversion recovery (FLAIR) MRI. The definition of 'extensive' resection has also been variable, with some studies reporting and evaluating the absolute volume of residual tumor $^{8}$ and others considering the percentage of the preoperative tumor volume that remained after surgery. ${ }^{6,22}$

Notably, a couple of recent studies suggest a possible role for neoadjuvant chemotherapy before surgical resection. ${ }^{39,40}$ In both reported cases, although contralateral involvement via the corpus callosum originally prohibited GTR, chemotherapy was able to shrink the tumors to such an extent as to make GTR possible. Studies of temozolomide for the treatment of low-grade glioma suggest that significant tumor shrinkage is possible with chemotherapy alone. ${ }^{15,41}$ It is unclear, however, how often chemotherapy can shrink a tumor enough to make GTR possible. The effect of the timing of surgery can and will be appreciated only when a prospective trial is performed, as was performed by van den Bent et al. to assess the timing of radiation therapy for the management of low-grade glioma. ${ }^{42}$

\section{ADVANCES IN NEUROSURGERY}

Advances in neurosurgical technology, including the introduction of preoperative and intraoperative brain mapping techniques and intraoperative image guidance, have made surgery for low-grade glioma increasingly safe and accessible. Perhaps more importantly, preoperative functional brain mapping has empowered surgeons to operate on patients who previously might not have been considered suitable candidates for surgery.

Historically, eloquent cortices have been identified on the basis of anatomical landmarks. In the context of brain tumors, however, these landmarks can be difficult to delineate, and this approach to identification cannot account for individual variability or plasticity-related changes. Preoperative brain mapping techniques, including functional MRI (fMRI) and magnetic 


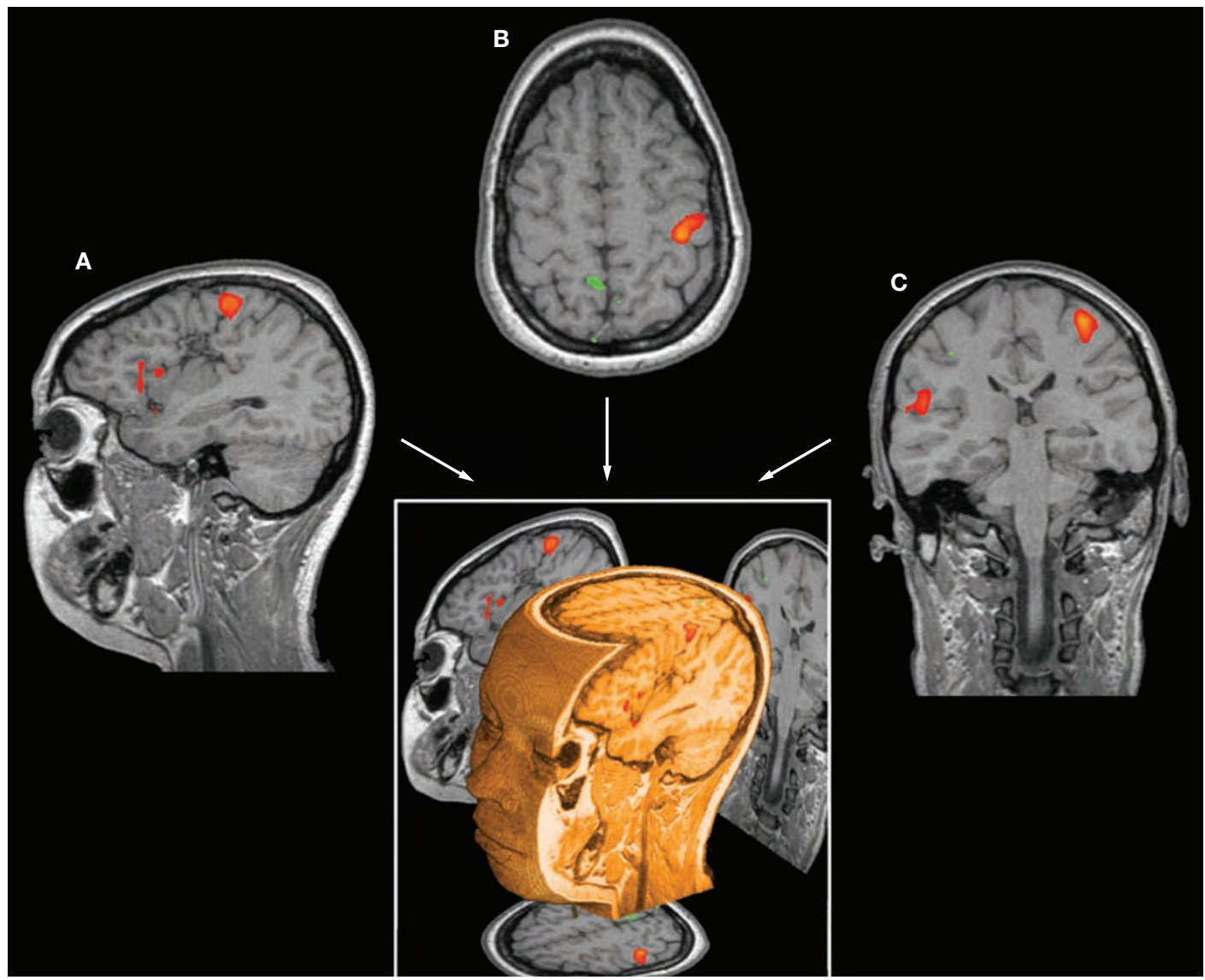

Figure 2 Functional MRI scan for preoperative planning. Rather than relying on anatomical landmarks for functional localization, functional MRI has enabled surgeons to precisely localize brain function and to use this information to plan for safer resection of low-grade glioma. (A) Axial, (B) sagittal and (C) coronal $\mathrm{T} 1 \mathrm{MRI}$ scans with overlying red areas of activation demonstrate the regions of the brain activated during right-hand motor activity. These images can be fused to create a three-dimensional rendering of functional anatomy to guide the surgeon and make surgery safer.

source imaging (MSI), have, therefore, revolutionized the assessment of patients with brain tumors. Preoperative maps help determine the need for intraoperative mapping, the best approach (or 'corridor') to tumor resection in order to spare eloquent cortices, and the limits of resection. Moreover, the use of preoperative maps can make intraoperative mapping safer and more efficient.

Blood-oxygen-level-dependent (BOLD) $\mathrm{fMRI},{ }^{43}$ a functional neuroimaging technique that maps the brain by detecting perfusionrelated changes that are coupled to cognitive tasks (and, therefore, to neuronal activity), has become the predominant functional neuroimaging technique (Figure 2). Recent technological advances, including increasing the magnetic resonance field strength, improved sequence and task design and selection, and superior analytical techniques, have made fMRI increasingly sensitive and reliable. ${ }^{44-49}$ Increasing the field strength provides improved signal-to-noise ratio and spatial resolution, and more-rapid imaging - a particularly important consideration in patients with low-grade glioma who might not tolerate prolonged imaging sessions. FitzGerald and colleagues found that fMRI was highly sensitive (81-92\%), but not very specific (0-53\%), for intraoperative identification of essential language areas. ${ }^{50}$ The low specificity (i.e. fMRI showed activation of areas that were not essential for linguistic tasks) suggests that fMRI could direct the surgeon to areas of interest so that it might be determined whether activated cortices are 'essential' or 'nonessential'- thereby obviating the need to 
map the entirety of the exposed cortex, but still necessitating intraoperative cortical mapping to guide resection. Nevertheless, the relationship between the use of fMRI maps and clinical outcomes remains uncharacterized. Moreover, the question of whether perfusion-related responses are adversely affected by an adjacent tumor has not yet been answered.

MSI fuses magnetoencephalography and MRI, projecting the extrapolated source localization of the magnetoencephalographic signals onto a co-registered anatomical MRI scan. As in other mapping techniques, MSI maps are generated by comparing 'activity' during a resting state with that detected during the performance of a specific task. Like fMRI, MSI correlates well with intraoperative maps. ${ }^{51,52}$ Moreover, MSI can be used to identify the $25-46 \%$ of patients with gliomas who are at risk of neurological deficit from surgical intervention. ${ }^{53-55}$ Patients with MSI signals within $5 \mathrm{~mm}$ of a tumor are considered to be at high risk of surgery-induced neurological deficit, those with activations between $6 \mathrm{~mm}$ and $10 \mathrm{~mm}$ from the tumor are slated for subtotal resection, and those with localizations greater than $10 \mathrm{~mm}$ from the tumor are deemed candidates for GTR. ${ }^{54}$

Diffusion tensor imaging (DTI) is an MRI technique that creates probabilistic maps of white matter pathways on the basis of voxel anisotropy, a measure of the preferential direction of diffusion of water. DTI can identify white matter pathways that have been either infiltrated or displaced by tumors. Preclinical studies suggest that DTI will have an increasingly important role in the planning of glioma surgery; the technique can identify white matter pathways that should be spared during surgery in order to ensure that functional cortices (as identified by other techniques) are not 'disconnected' from their respective projection areas. ${ }^{56}$ Kamada and colleagues verified that preoperative cortical and subcortical maps generated by a combination of fMRI, MSI and DTI are predictive and can be confirmed by intraoperative cortical and subcortical stimulation mapping. ${ }^{57}$

In the operating room, technological advances have made cortical and subcortical stimulation mapping easier to implement, making these techniques a more routine part of neurosurgery. In view of its reliability in predicting postoperative outcomes, stimulation mapping is considered to be the gold standard for neurosurgical brain mapping. In this approach, the brain is mapped by either activating the tissue of interest, as in motor mapping, or by temporarily inducing deficits, as in language mapping. To evaluate the effect of stimulation mapping on the efficacy and safety of surgery for low-grade glioma, Duffau and colleagues compared a retrospective series of 100 patients who were operated on without guidance from intraoperative mapping (between 1985 and 1996) and a prospective series of 122 patients who were operated on with concurrent use of electrocortical stimulation mapping (between 1996 and 2003). ${ }^{58}$ The latter group had a significantly lower rate of severe permanent deficits (6.5\% vs $17 \%)$, a higher rate of GTR and subtotal resections ( $25 \%$ and $51 \%$ vs $6 \%$ and $37 \%$, respectively), and a survival advantage, compared with the former group. ${ }^{58}$ Moreover, the authors found that the definition of 'operability' changed considerably between the two series: the percentage of tumors operated on within eloquent cortices increased from $35 \%$ to $62 \%$. Stimulation of subcortical white matter tracts has also become an increasingly important adjunct to intraoperative mapping, permitting the surgeon to spare descending pathways and predict postoperative morbidity. ${ }^{59}$ Patients in whom subcortical motor tracts are identified in the resected areas are significantly more likely to experience temporary or permanent motor deficits than those in whom subcortical motor pathways cannot be identified in these areas. ${ }^{60}$

In addition to stimulation mapping, advanced intraoperative image guidance techniques can now provide a highly accurate real-time threedimensional 'road map' to an individual patient's brain, which enables surgeons to operate more safely in the face of distorted anatomy. These road maps are created by registering anatomical or artificial landmarks (fiducials) on a high-resolution preoperative MRI scan of the patient's head and pairing them with corresponding positions on the patient's head after it is positioned for surgery. Current frameless stereotactic neuronavigation devices use optical triangulation or low-frequency electromagnetic fields to determine the location of an instrument within the operative field. Intraoperative image guidance provides ongoing feedback with respect to regional anatomical relationships, location of normal tissues that must be preserved, and extent of resection. This feedback 
during surgery can be particularly useful for low-grade glioma, in which the delineation between tumor and surrounding white matter is indistinct. Lumenta et al. described a series of 40 patients (21 of whom had glial neoplasms) who underwent resection of deep-seated intracerebral lesions guided by neuronavigation and cortical mapping. Complete resection was possible in all but three of the patients, and permanent neurological deficits were only noted in two. ${ }^{61}$ Although excellent for initial planning, the reliability of maps from preoperative MRI for intraoperative guidance decreases during surgery owing to 'brain shift', a phenomenon attributable to tumor removal, ongoing cerebrospinal fluid loss, and brain edema. ${ }^{62,63}$

The limitations of intraoperative image guidance have been partially overcome with the advent of intraoperative magnetic resonance systems that provide real-time imaging of the brain in its intraoperative position. Black and colleagues highlighted the important contribution of intraoperative MRI to successful tumor resection with the observation that, in over one-third of cases, the surgeon's judgment of the extent of GTR was deemed incorrect by intraoperative MRI, thus prompting further surgical resection. ${ }^{64}$ Similarly, Wirtz et al. found that implementation of intraoperative MRI resulted in a significantly higher rate of GTR (67\% compared with 38\% without the use of intraoperative MRI), which was associated with a significant increase in survival times. ${ }^{65}$ Claus and co-workers found that patients who underwent surgical resection of low-grade gliomas with concurrent intraoperative MRI guidance had better 1-year, 2-year and 5-year survival rates than those reported in the CBTRUS. ${ }^{66}$ The authors also suggested a possible association between GTR using intraoperative MRI guidance and prolonged PFS and OS (although this correlation did not achieve significance). Further studies of this topic are warranted, especially in light of the ongoing interest in defining the effect of GTR on outcomes in low-grade glioma.

Notwithstanding the technological advances that have made surgical resection a viable option in a greater number of patients with low-grade glioma, it is still recognized that GTR might not be possible in all cases. The radiographic appearance of a low-grade glioma necessarily dictates its amenability to resection. Low-grade gliomas might involve critical structures, including the corpus callosum, thalamus or basal ganglia, might be well circumscribed or in filtrative, and might be unilateral or bilateral. Predictors of incomplete tumor resection include diffuse tumor margin on T2-weighted MRI, oligodendroglioma or oligoastrocytoma histopathological tumor type, large tumor volume, and tumor involving the corpus callosum, corticospinal tract, insular lobe, middle cerebral artery, motor cortex, optic radiation, visual cortex, or basal ganglia. ${ }^{67}$ In general, unilateral, well-circumscribed, cortexbased lesions are ideal candidates for resection. Nonetheless, recent reports suggest that tumors that were once considered unresectable, owing to involvement of the corpus callosum, insula or eloquent language cortices, can now undergo resective procedures without the risk of sequelae being increased. ${ }^{68,69}$

\section{FUTURE TRIALS}

Given the advances in modern neurosurgical and neuroimaging techniques, which permit safer surgical resections and more-accurate assessment of the extent of resection than was previously possible, neurosurgeons and neuro-oncologists might finally be in a position to accurately explore the extent to which aggressive resection improves the prognosis of low-grade glioma.

Planning a trial to compare conservative and aggressive surgical management is complex and poses several challenges. The trial should ideally be randomized, but ethical concerns have limited this approach. A randomized trial was proposed to the American College of Surgeons Oncology Group (ACOSOG), in which patients with incidental low-grade glioma (or those well controlled with a single antiepileptic agent after a single seizure) would be randomly allocated to either biopsy or surgical resection. This trial was, however, rejected over concerns that the literature might already contain sufficient evidence to support resection. Instead of a randomized design, a trial must, therefore, be designed with a prospective cohort construction. To obtain adequate accrual and followup, such a trial would require the enrollment of at least 1,100 patients, who would have to be observed for approximately 10 years (or until death).${ }^{70}$ In light of the low incidence of lowgrade glioma $(2,000-3,000$ cases per year in the US), enrollment of a sufficient number of patients would necessitate multi-institutional-if not multinational-cooperation. To reduce 
institutional bias, data must be collected and analyzed at a central location. To ensure the validity of the trial, the extent of resection would have to be evaluated objectively by unbiased investigators (i.e. investigators not involved with patient care and blinded to patient outcomes) who would use volumetric measurements from T2-weighted or FLAIR MRI sequences. Primary end points should include OS, PFS, and QOL (including assessments of neurocognitive function). Pre-planned statistical analyses should account for known prognostic factors, including age, preoperative tumor size, and histopathological subtype (including $1 \mathrm{p}$ and $19 \mathrm{q}$ deletion status). Moreover, analyses should take into account factors that limit the success of GTR, including diffuse tumor margin on T2-weighted MRI and involvement of eloquent areas (e.g. visual cortex and motor cortex). Finally, analyses must account for adjunctive therapies, including fractionated radiotherapy and chemotherapy. Interim analyses should be planned in case either biopsy or surgical resection proves to be superior on early analysis. Clearly, the coordination, data collection and statistical analysis that would be involved in such a trial would be technically difficult, but this method might be the only means of definitively answering the question regarding the role of surgical resection in the management of low-grade glioma.

\section{CONCLUSIONS}

Although a 'wait-and-see' policy might not adversely affect patients with low-grade glioma, the emergence of novel therapeutic strategies has increased the importance of obtaining tissue for both histological and cytogenetic characterization, so as to optimize patient management. Although the benefit of tissue acquisition by either biopsy or cytoreductive surgery seems indisputable, the precise role of surgical resection-especially in patients without mass effect or symptoms of intracranial hypertensionremains unclear. The literature provides modest evidence that surgery improves patient outcomes by reducing tumor burden. Nonetheless, it is not possible to devise a prescribed treatment algorithm on the basis of the current level of evidence. Until prospective trials have been conducted and analyzed, neuro-oncologists and neurosurgeons must continue to individualize the evaluation and treatment of low-grade glioma on the basis of symptomatology, patient and tumor characteristics, and patient preference.

\section{KEY POINTS}

- Approximately 2,000-3,000 low-grade gliomas are diagnosed in the US every year, resulting in considerable morbidity and inevitable death

- Management options for low-grade glioma include observation, radiotherapy, chemotherapy and surgical resection; the role of surgical resection is particularly controversial

- The literature provides modest evidence that surgery improves outcomes by reducing tumor burden, but there is a paucity of class I evidence

- Advances in neurosurgical and neuroimaging techniques have made surgical resection of low-grade gliomas a safe option for an increasing number of patients

- In light of recent advances, the time might be right to effectively and accurately assess the effect of aggressive surgical resection on the prognosis of low-grade glioma

\section{References}

1 Wallner KE et al. (1988) Treatment results of juvenile pilocytic astrocytoma. J Neurosurg 69: 171-176

2 Central Brain Tumor Registry of the United States. Statistical report: primary brain tumors in the United States, 1998-2002. [http://www.cbtrus.org/ reports//2005-2006/2006report.pdf]

3 Shaw E et al. (2002) Prospective randomized trial of low- versus high-dose radiation therapy in adults with supratentorial low-grade glioma: initial report of a North Central Cancer Treatment Group/Radiation Therapy Oncology Group/Eastern Cooperative Oncology Group study. J Clin Oncol 20: 2267-2276

4 Duffau H and Capelle L (2004) Preferential brain locations of low-grade gliomas. Cancer 100: 2622-2626

5 Piepmeier J et al. (1996) Variations in the natural history and survival of patients with supratentorial lowgrade astrocytomas. Neurosurgery 38: 872-878

6 Pignatti F et al. (2002) Prognostic factors for survival in adult patients with cerebral low-grade glioma. $J$ Clin Oncol 20: 2076-2084

7 Leighton C et al. (1997) Supratentorial low-grade glioma in adults: an analysis of prognostic factors and timing of radiation. J Clin Oncol 15: 1294-1301

8 Berger MS et al. (1994) The effect of extent of resection on recurrence in patients with low grade cerebral hemisphere gliomas. Cancer 74: 1784-1791

9 Mariani Let al. (2004) The impact of tumour volume and surgery on the outcome of adults with supratentorial WHO grade II astrocytomas and oligoastrocytomas. Acta Neurochir (Wien) 146: 441-448

10 Karim AB et al. (1996) A randomized trial on doseresponse in radiation therapy of low-grade cerebral glioma: European Organization for Research and Treatment of Cancer (EORTC) study 22844. Int J Radiat Oncol Biol Phys 36: 549-556

11 Pallud J et al. (2006) Prognostic value of initial magnetic resonance imaging growth rates for World Health Organization grade II gliomas. Ann Neurol 60: 380-383 
12 Cairncross JG and Laperriere NJ (1989) Low-grade glioma. To treat or not to treat? Arch Neurol 46: 1238-1239

13 Kondziolka D et al. (1993) Unreliability of contemporary neurodiagnostic imaging in evaluating suspected adult supratentorial (low-grade) astrocytoma. J Neurosurg 79: $533-536$

14 Scott JN et al. (2002) How often are nonenhancing supratentorial gliomas malignant? A population study. Neurology 59: 947-949

15 Pace A et al. (2003) Temozolomide chemotherapy for progressive low-grade glioma: clinical benefits and radiological response. Ann Oncol 14: 1722-1726

16 Spampinato MV et al. (2007) Cerebral blood volume measurements and proton MR spectroscopy in grading of oligodendroglial tumors. AJR Am J Roentgenol 188: 204-212

17 Law M et al. (2003) Glioma grading: sensitivity, specificity, and predictive values of perfusion MR imaging and proton MR spectroscopic imaging compared with conventional MR imaging. AJNR Am J Neuroradiol 24: 1989-1998

18 Floeth FW and Stummer W (2005) The value of metabolic imaging in diagnosis and resection of cerebral gliomas. Nat Clin Pract Neurol 1: 62-63

19 Keles GE et al. (2001) Low-grade hemispheric gliomas in adults: a critical review of extent of resection as a factor influencing outcome. J Neurosurg 95: 735-745

20 Recht LD et al. (1992) Suspected low-grade glioma: is deferring treatment safe? Ann Neurol 31: 431-436

21 Reijneveld JC et al. (2001) Cognitive status and quality of life in patients with suspected versus proven lowgrade gliomas. Neurology 56: 618-623

22 van Veelen ML et al. (1998) Supratentorial low grade astrocytoma: prognostic factors, dedifferentiation, and the issue of early versus late surgery. J Neurol Neurosurg Psychiatry 64: 581-587

23 Jackson RJ et al. (2001) Limitations of stereotactic biopsy in the initial management of gliomas. Neuro Oncol 3: 193-200

24 Kondziolka D and Lunsford LD (1999) The role of stereotactic biopsy in the management of gliomas. J Neurooncol 42: 205-213

25 Yu X et al. (2000) Stereotactic biopsy for intracrania space-occupying lesions: clinical analysis of 550 cases. Stereotact Funct Neurosurg 75: 103-108

26 Aker FV et al. (2005) Accuracy and diagnostic yield of stereotactic biopsy in the diagnosis of brain masses: comparison of results of biopsy and resected surgical specimens. Neuropathology 25: 207-213

27 Hall WA (1998) The safety and efficacy of stereotactic biopsy for intracranial lesions. Cancer 82: 1749-1755

28 McGirt MJ et al. (2003) MRI-guided stereotactic biopsy in the diagnosis of glioma: comparison of biopsy and surgical resection specimen. Surg Neurol 59: 277-281

29 Feiden W et al. (1991) Accuracy of stereotactic brain tumor biopsy: comparison of the histologic findings in biopsy cylinders and resected tumor tissue. Neurosurg Rev 14: 51-56

30 Chandrasoma PT et al. (1989) Stereotactic biopsy in the diagnosis of brain masses: comparison of results of biopsy and resected surgical specimen. Neurosurgery 24: 160-165

31 McGirt MJ et al. (2005) Independent predictors of morbidity after image-guided stereotactic brain biopsy: a risk assessment of 270 cases. J Neurosurg 102: 897-901

32 Keles GE et al. (2004) Low-grade gliomas: astrocytoma, oligodendroglioma, and mixed gliomas. In Youmans Neurological Surgery, 950-968 (Eds Winn HR and Youmans JR) Philadelphia: WB Saunders
33 Gunnarsson T et al. (2002) Surgical treatment of patients with low-grade astrocytomas and medically intractable seizures. Acta Neurol Scand 105: 289-292

34 Nakamura M et al. (2000) Analysis of prognostic and survival factors related to treatment of low-grade astrocytomas in adults. Oncology 58: 108-116

35 Lo SS et al. (2001) Does the extent of surgery have an impact on the survival of patients who receive postoperative radiation therapy for supratentorial low-grade gliomas? Int J Cancer 96 (Suppl 1): 71-78

36 Bauman Get al. (1999) Pretreatment factors predict overall survival for patients with low-grade glioma: a recursive partitioning analysis. Int J Radiat Oncol Biol Phys 45: 923-929

37 Lote Ket al. (1997) Survival, prognostic factors, and therapeutic efficacy in low-grade glioma: a retrospective study in 379 patients. J Clin Oncol 15: 3129-3140

38 Teixidor P et al. (2006) Assessment of verbal working memory before and after surgery for low-grade glioma J Neurooncol 81: 305-313

39 Duffau Het al. (2006) Radical surgery after chemotherapy: a new therapeutic strategy to envision in grade II glioma. J Neurooncol 80: 171-176

40 Voloschin AD et al. (2005) Neoadjuvant temozolomide followed by complete resection of a $1 p$ - and $19 q-$ deleted anaplastic oligoastrocytoma: case study. Neuro Oncol 7: 97-100

41 van den Bent MJ et al. (2003) Phase II study of firstline chemotherapy with temozolomide in recurrent oligodendroglial tumors: the European Organization for Research and Treatment of Cancer Brain Tumor Group study 26971. J Clin Oncol 21: 2525-2528

42 van den Bent MJ et al. (2005) Long-term efficacy of early versus delayed radiotherapy for low-grade astrocytoma and oligodendroglioma in adults: the EORTC 22845 randomised trial. Lancet 366: 985-990

43 Belliveau JW et al. (1991) Functional mapping of the human visual cortex by magnetic resonance imaging. Science 254: 716-719

44 Roessler Ket al. (2005) Evaluation of preoperative high magnetic field motor functional MRI (3 Tesla) in glioma patients by navigated electrocortical stimulation and postoperative outcome. J Neurol Neurosurg Psychiatry 76: 1152-1157

45 Kruger G et al. (2001) Neuroimaging at 1.5 T and 3.0 T: comparison of oxygenation-sensitive magnetic resonance imaging. Magn Reson Med 45: 595-604

46 Roux FE et al. (2003) Language functional magnetic resonance imaging in preoperative assessment of language areas: correlation with direct cortical stimulation. Neurosurgery 52: 1335-1345

47 Pouratian N et al. (2002) Utility of preoperative functional magnetic resonance imaging for identifying language cortices in patients with vascular malformations. J Neurosurg 97: 21-32

48 Roux FE et al. (1999) Usefulness of motor functional $\mathrm{MRI}$ correlated to cortical mapping in rolandic lowgrade astrocytomas. Acta Neurochir (Wien) 141: 71-79

49 Pouratian N and Bookheimer SY (2006) Clinical challenges of fMRI. In fMRI: Basic Principles and Clinical Applications, 99-126 (Eds Mohamed F and Faro S) New York: Springer

50 FitzGerald DB et al. (1997) Location of language in the cortex: a comparison between functional MR imaging and electrocortical stimulation. AJNR Am J Neuroradiol 18: 1529-1539

51 Gallen CC et al. (1995) Presurgical localization of functional cortex using magnetic source imaging. J Neurosurg 82: 988-994 
52 Schiffbauer Het al. (2002) Preoperative magnetic source imaging for brain tumor surgery: a quantitative comparison with intraoperative sensory and motor mapping. J Neurosurg 97: 1333-1342

53 Schiffbauer $\mathrm{H}$ et al. (2001) Functional activity within brain tumors: a magnetic source imaging study. Neurosurgery 49: 1313-1320

54 Ganslandt O et al. (2004) Magnetic source imaging supports clinical decision making in glioma patients. Clin Neurol Neurosurg 107: 20-26

55 Grummich P et al. (2006) Combining fMRI and MEG increases the reliability of presurgical language localization: a clinical study on the difference between and congruence of both modalities. Neuroimage 32: 1793-1803

56 Berman Jl et al. (2004) Diffusion-tensor imagingguided tracking of fibers of the pyramidal tract combined with intraoperative cortical stimulation mapping in patients with gliomas. J Neurosurg 101: 66-72

57 Kamada Ket al. (2007) Visualization of the frontotemporal language fibers by tractography combined with functional magnetic resonance imaging and magnetoencephalography. J Neurosurg 106: 90-98

58 Duffau Het al. (2005) Contribution of intraoperative electrical stimulations in surgery of low grade gliomas: a comparative study between two series without (1985-96) and with (1996-2003) functional mapping in the same institution. J Neurol Neurosurg Psychiatry 76: 845-851

59 Duffau H et al. (2002) Intraoperative mapping of the subcortical language pathways using direct stimulations. An anatomo-functional study. Brain 125: 199-214

60 Keles GE et al. (2004) Intraoperative subcortical stimulation mapping for hemispherical perirolandic gliomas located within or adjacent to the descending motor pathways: evaluation of morbidity and assessment of functional outcome in 294 patients. J Neurosurg 100: 369-375

61 Lumenta CB et al. (1997) Three-dimensional computer-assisted stereotactic-guided microneurosurgery combined with cortical mapping of the motor area by direct electrostimulation. Minim Invasive Neurosurg 40: 50-54
62 Maurer CR Jr et al. (1998) Investigation of intraoperative brain deformation using a 1.5-T interventional MR system: preliminary results. IEEE Trans Med Imaging 17: 817-825

63 Nimsky C et al. (2001) Intraoperative compensation for brain shift. Surg Neurol 56: 357-364

64 Black PM et al. (1999) Craniotomy for tumor treatment in an intraoperative magnetic resonance imaging unit. Neurosurgery 45: 423-431

65 Wirtz CR et al. (2000) Clinical evaluation and followup results for intraoperative magnetic resonance imaging in neurosurgery. Neurosurgery 46: 1112-1120

66 Claus EB et al. (2005) Survival rates in patients with low-grade glioma after intraoperative magnetic resonance image guidance. Cancer 103: 1227-1233

67 Talos IF et al. (2006) Supratentorial low-grade glioma resectability: statistical predictive analysis based on anatomic MR features and tumor characteristics. Radiology 239: 506-513

68 Duffau Het al. (2004) Surgical removal of corpus callosum infiltrated by low-grade glioma: functional outcome and oncological considerations. J Neurosurg 100: $431-437$

69 Bello Let al. (2007) Intraoperative subcortical language tract mapping guides surgical removal of gliomas involving speech areas. Neurosurgery 60: 67-82

70 Laws ER (2001) Resection of low-grade gliomas. J Neurosurg 95: 731-732

71 Laws ER Jr et al. (1984) Neurosurgical management of low-grade astrocytoma of the cerebral hemispheres. J Neurosurg 61: 665-673

72 Shaw EG et al. (1989) Radiation therapy in the management of low-grade supratentorial astrocytomas. J Neurosurg 70: 853-861

73 Philippon JH et al. (1993) Supratentorial low-grade astrocytomas in adults. Neurosurgery 32: 554-559

74 Shibamoto Y et al. (1993) Supratentorial low-grade astrocytoma. Correlation of computed tomography findings with effect of radiation therapy and prognostic variables. Cancer 72: 190-195

75 Scerrati M et al. (1996) Prognostic factors in low grade (WHO grade II) gliomas of the cerebral hemispheres: the role of surgery. J Neurol Neurosurg Psychiatry 61: 291-296
Acknowledgments

Désirée Lie, University of California, Irvine, CA, is the author of and is solely responsible for the content of the learning objectives, questions and answers of the Medscapeaccredited continuing medical education activity associated with this article.

\section{Competing interests} The authors declared no competing interests. 